\title{
Cultura empresarial en las pymes del municipio de Tamazula de Gordiano, Jalisco (México)*
}

\author{
[Versión en Castellano]
}

Business Culture in SMEs in the Municipality of

Tamazula de Gordiano, Jalisco (Mexico)

Cultura empresarial nas PME do município de

Tamazula de Gordiano, Jalisco (México)

Recibido el 3 de mayo, 2019. Aceptado el 6 de diciembre, 2019.

- Para citar este artículo:

Hinojosa-Gómez, Carlos-Adolfo;

Limón-Villegas Edgar-Samid;

Navarrete-Cova Citlali (2020)

Cultura empresarial en las pymes

del municipio de Tamazula de

Gordiano, Jalisco (México).

Ánfora, 27(49), 143-172.

https://doi.org/10.30854/anfv27.n49.2020.743

Universidad Autónoma de

Manizales. ISSN 0121-6538/

e-ISSN 2248-6941.

CC BY-NC-SA 4.0
Carlos-Adolfo Hinojosa-Gómez ${ }^{* *}$

https://orcid.org/0000-0002-1897-0019

México

Edgar-Samid Limón-Villegas***

https://orcid.org/0000-0003-2191-316X

México

Citlali Navarrete-Cova****

https://orcid.org/0000-0001-9996-1319

México
* Tamazula de Gordiano: municipio ubicado en la región sureste del Estado de Jalisco, occidente de México. Se caracteriza por grandes extensiones de cultivos de caña de azúcar, jitomate, maíz y frutales como aguacate. Destaca el Ingenio Tamazula que produce azúcar y alcohol de caña (Gobierno de Jalisco, 2019). Los investigadores declaran que no hubo conflictos de intereses en la ejecución del proyecto de investigación.

** Doctor. Profesor-investigador de tiempo completo, miembro del cuerpo académico: ITJMMPH-CA-9 Centro de Estudios para el Aprendizaje Innovador (C.E.P.A.I.) del Tecnológico Nacional de México/ITJMMPYH, campus Tamazula, Jalisco México. Correo electrónico: adolfo.hinojosa@tamazula.tecmm.edu.mx

*** Profesor-investigador de tiempo completo, miembro del cuerpo académico: ITJMMPH-CA-9 Centro de Estudios para el Aprendizaje Innovador (C.E.P.A.I.), del Tecnológico Nacional de México/ITJMMPYH, campus Tamazula, Jalisco México. Correo electrónico: samid.limon@tamazula.tecmm.edu.mx

**** Profesor-investigador de tiempo completo, miembro del cuerpo académico: ITJMMPH-CA-9 Centro de Estudios para el Aprendizaje Innovador (C.E.P.A.I.), del Tecnológico Nacional de México/ITJMMPYH, campus Tamazula, Jalisco México. Correo electrónico: citlali.navarrete@tamazula.tecmm.edu.mx 


\section{Resumen}

Objetivo: examinar la cultura empresarial de las pequeñas y medianas empresas del municipio de Tamazula de Gordiano, México. Metodología: la investigación se realizó con un enfoque cuantitativo y el método fue de tipo cuasi experimental transeccional y exploratorio. Cuasi experimental porque se llevó a cabo sin manipular variables y se basó en la comunidad de pymes originarias del municipio de Tamazula de Gordiano; transeccional exploratorio, porque se comenzó a conocer sobre el concepto de cultura organizacional y exploratoria, que se aplica a problemas de investigación poco estudiados en dicha comunidad. Se aplicaron encuestas a los gerentes o dueños de 35 pequeñas y medianas empresas del sector privado (Pymes), utilizando como instrumento el cuestionario de "diagnóstico empresarial" desarrollado por el Instituto para el Desarrollo y la Innovación en la Tecnología para la Pequeña y Mediana Empresa (IDITpyme), de la Universidad de Guadalajara, considerando el área de cultura empresarial. Resultados: la cultura empresarial de las pymes de Tamazula de Gordiano no distingue o separa el capital de la empresa y el patrimonio familiar; solo una minoría cuenta con una filosofía organizacional definida y conocida por sus miembros. La mayoría de los gerentes o dueños consideran a las alianzas estratégicas con poca o sin importancia alguna para el logro de los objetivos organizacionales y tienen una posición reactiva frente a posibles amenazas externas. Considerando las características anteriormente descritas, se infiere que la cultura empresarial de las pymes, de acuerdo con la clasificación de Robbins es débil. La cultura empresarial que caracteriza las pymes, objeto de estudio, supone un desempeño inferior, lo que las coloca en una situación de desventaja frente a las grandes empresas que cuentan con una cultura fuerte y que, además, las pone en grave riesgo de permanencia ante una situación económica local y nacional cada vez más compleja, cambiante y competitiva. Conclusiones: se concluye que esta cultura empresarial débil, es similar en las pymes de los distintos municipios de México y otros países de Latinoamérica, dadas las similitudes culturales que persisten y coexisten en esta zona geográfica del mundo.

Palabras-clave: Cultura empresarial; Cultura organizacional; Diagnóstico; Pymes; Valores.

\section{Abstract}

Objective: to examine the business culture of small and medium-sized companies in the municipality of Tamazula de Gordiano, Mexico. Methodology: the research was carried out with a quantitative approach and the method was quasi-experimental, transectional and exploratory. It was quasi experimental because it was carried out 
without manipulating variables and was based on the community of SMEs from the municipality of Tamazula de Gordiano; exploratory transectional, because the concept of exploratory and organizational culture began to be understood, which is applied to research, problems that are little studied in said community. Surveys were applied to the managers or owners of 35 small and medium-sized private sector companies (SMEs), using the "business diagnosis" questionnaire as an instrument, developed by the Institute for Development and Innovation in Technology for Small and Mediumsized Enterprises (IDITpyme), from the University of Guadalajara, considering the area of business culture. Results: the business culture of SMEs in Tamazula de Gordiano does not distinguish or separate the capital of the company from family assets; only a minority has an organizational philosophy defined and known by its members. Most managers or owners consider strategic alliances of little or no importance to the achievement of organizational objectives and are reactive in the face of possible external threats. Considering the characteristics described above, it is inferred that the business culture of SMEs, according to the Robbins classification, is weak. The business culture that characterizes SMEs, the object of the study, implies a lower performance, which places them at a disadvantage compared to large companies that have a strong culture and, furthermore, puts them at serious risk of remaining vulnerable in the face of an increasingly complex, changing and competitive local and national economic situation. Conclusions: It is concluded that this weak business culture is similar in SMEs in the different municipalities of Mexico and other Latin American countries, given the cultural similarities that persist and coexist in this geographical area of the world.

Keywords: Corporate culture; Organizational culture; Diagnosis; SMEs; Values.

\section{Resumo}

Objetivos: examinar a cultura de negócios de pequenas e médias empresas no município de Tamazula de Gordiano, México. Metodologia: a pesquisa foi realizada com abordagem quantitativa e o método foi quase experimental, transecional e exploratório. Quase experimental, porque foi realizada sem manipulação de variáveis e baseou-se na comunidade de PMEs do município de Tamazula de Gordiano; transecional exploratória, porque se tornou conhecido o conceito de cultura exploratória e organizacional, aplicada a problemas de pesquisa pouco estudados nessa comunidade. As pesquisas foram aplicadas aos gestores ou proprietários de 35 pequenas e médias empresas do setor privado (PME), utilizando como instrumento o questionário "diagnóstico de negócios" desenvolvido pelo Instituto de Desenvolvimento e Inovação em Tecnologia para Pequenas e Médias Empresas (IDITpyme), da Universidade de Guadalajara, considerando a área de cultura empresarial. Resultados: a cultura empresarial das PME 
em Tamazula de Gordiano não distingue ou separa o capital da empresa e os ativos da família; Somente uma minoria tem uma filosofia organizacional definida e conhecida por seus membros. A maioria dos gerentes ou proprietários considera alianças estratégicas de pouca ou nenhuma importância para a consecução dos objetivos organizacionais e é reativa diante de possíveis ameaças externas. Considerando as características descritas acima, infere-se que a cultura de negócios das PMEs, de acordo com a classificação de Robbins, é fraca. A cultura de negócios que caracteriza as PMEs, objeto do estudo, implica um desempenho inferior, o que as coloca em desvantagem em comparação às grandes empresas que possuem uma cultura forte e, além disso, as colocam em sério risco de permanência antes uma situação econômica local e nacional cada vez mais complexa, mutável e competitiva. Conclusões: Conclui-se que essa fraca cultura comercial é semelhante nas PMEs dos diferentes municípios do México e de outros países da América Latina, dadas as semelhanças culturais que persistem e coexistem nessa área geográfica do mundo.

Palavras-chave: Cultura de negócios; Cultura organizacional; Diagnóstico; PME; Valores.

\section{Introducción}

La cultura en términos genéricos se refiere a los valores, creencias y tradiciones de una sociedad; estos valores se reproducen también dentro de las organizaciones haciendo que sus integrantes se comporten de una u otra forma. La cultura es intrínseca a cualquier tipo de organización, es la personalidad que las hace originales, es como la huella digital en las personas, irrepetible. Por ende, las pequeñas y medianas empresas (pymes), como organizaciones, tienen también una forma única de comportarse, de ver las cosas y de reaccionar ante los problemas que enfrentan. Al conjunto de estas singularidades se les denomina "cultura empresarial, organizacional o corporativa".

Una de las principales características en las organizaciones del siglo XXI, es su dinamismo, por la forma en que las organizaciones resuelven los problemas y cómo buscan nuevas oportunidades de negocio, que les permita evolucionar y adaptarse exitosamente a los retos de la nueva realidad empresarial. En este contexto, las pymes sin lugar a duda, son de vital importancia para el desarrollo económico de México a causa de su contribución económica combinada, que suma el 36\% del Producto Interno Bruto (PIB) y el 32\% del empleo (Secretaría de Economía, 2010). 
El reconocido sociólogo chileno, Darío Rodríguez (2016), explica que el tema de la cultura organizacional toma relevancia a partir de la década de 1980, con el surgimiento del modelo japonés como una forma de organización culturalmente basada, la globalización, el reemplazo de la generación de "baby-boomers" por la "Y", motivaron a entender las particularidades de la cultura como modelo de ser en las organizaciones, siendo la presente década del siglo XXI destacada para el estudio de las culturas laborales.

En el contexto latinoamericano se han realizado algunas investigaciones sobre cultura organizacional o empresarial en la pequeña y mediana empresa. En Colombia, Marulanda, López y Castellanos. (2016), realizaron un trabajo de investigación con el objetivo de exponer la relación entre la cultura organizacional y las buenas prácticas para la gestión del conocimiento (G. C.) en las pymes de Colombia, para formular acciones que permitan su crecimiento (p. 163), concluyendo que la cultura organizacional influye positivamente en el impacto que tienen las buenas prácticas para la gestión del conocimiento, debido a los esfuerzos hechos por las compañías en el reconocimiento de la importancia de la G. C. (Marulanda et al., p. 174).

En México los autores Gómez, Villareal y Villareal (2014), publicaron un artículo titulado, "la cultura empresarial y su relación con los estilos de aprendizaje en la industria de la celulosa, cartón y papel en México", en el que hacen un diagnóstico de la cultura empresarial dominante, los estilos de aprendizaje preponderantes y la relación de ambos conceptos, llegando al conclusión de que "la cultura dominante en el sector estudiado, es la cultura de mercado", y agregan que "una empresa tiene una cultura dominante, que es acompañada en diferentes intensidades de las características de las otras tres culturas" (...) adhocrática, de clan y jerárquica (Gómez et al., p. 22).

El presente trabajo de investigación utiliza como fuente de datos para realizar el examen, empresas categorizadas como pymes originarias y localizadas dentro del municipio de Tamazula de Gordiano, Jalisco, México, según datos del Instituto Nacional de Estadística y Geografía, INEGI (2000), por medio de entrevistas aplicadas a los gerentes o dueños de las empresas, utilizando como instrumento el cuestionario de diagnóstico empresarial desarrollado por el Instituto para el Desarrollo y la Innovación en la Tecnología para la Pequeña y Mediana Empresa (IDITpPyme), de la Universidad de Guadalajara, considerando el área de cultura empresarial.

El objetivo es el de examinar los aspectos más significativos de la cultura empresarial en las pymes, como una primera aproximación para entender la situación cultural de las mismas y proponer en futuras investigaciones acciones concretas que mejoren su desarrollo y competitividad. Los objetivos específicos que se ha fijado este estudio son: 
1. Seleccionar a las pequeñas y medianas empresas, con base al número de empleados, originarias y localizadas en el municipio de Tamazula de Gordiano, Jalisco.

2. Analizar los principales elementos de la cultura organizacional en las empresas seleccionadas objeto de estudio.

3. Categorizar la cultura empresarial de las pymes del municipio de Tamazula.

\section{Hipótesis}

La cultura empresarial de las pymes el municipio de Tamazula de Gordiano Jalisco se caracteriza por ser débil, sin una filosofía organizacional establecida ni compartida entre los integrantes y con una forma reactiva de afrontar los problemas del entorno.

\section{Antecedentes y aportaciones teóricas}

Primero se definen los conceptos más relevantes para esta investigación y luego se detallan los antecedentes y aportaciones teóricas sobre el tema.

\section{Conceptos.}

\section{Análisis.}

"Distinción y separación de las partes de algo para conocer su composición”. Otra acepción dice que es el "estudio detallado de algo, especialmente de una obra o de un escrito” (Real Academia Española, 2018).

\section{Análisis Institucional.}

De acuerdo con Franklin y Krieger (2011), “el análisis institucional tiene el propósito de descubrir el inconsciente colectivo de las organizaciones, los grupos y la sociedad en general" (p. 143).

\section{Alianzas estratégicas.}

Se definen como: “acuerdos de cooperación que pueden tener un carácter local, internacional e incluso global, para establecer convenios con otras compañías con el fin de realizar una nueva operación, buscando compartir costos, riesgos y beneficios al incursionar en el desarrollo de negocios" (Benavides-Pañeda, 2004, pp. 30-31).

\section{Cultura organizacional.}

"Conjunto de premisas básicas sobre las que se construye el decidir organizacional” (Rodríguez, 2016, p. 152). 


\section{Cultura dominante.}

"Expresa los valores fundamentales que comparte la mayoría de una organización. Cuando se habla de la cultura de una organización, se hace referencia a su cultura dominante" (Robbins y Judge, 2009, p. 553).

\section{Cultura fuerte.}

Aquella donde los valores fundamentales se comparten con intensidad y en forma extensa (Robbins y Judge, 2013).

\section{Cultura débil.}

Cultura en donde no se asumen los valores, sino que se conciben como impuestos (Robbins y Judge, 2013).

\section{Diagnóstico.}

"Proceso de acercamiento gradual al conocimiento analítico de un hecho o problema, que permite destacar los elementos más significativos de su composición y funcionamiento, para derivar acciones de ajuste y/o desarrollo orientadas a optimizarlo" (Franklin, 2014, p.385).

\section{Pyme.}

Acrónimo de pequeña y mediana empresa.

\section{Valor.}

"En las corrientes más actuales valor es aquello que orienta a la acción” (Garza-Treviño, 2004, p. 389).

\section{Valores.}

"Los valores de la empresa deberán ser los mapas que dirijan todos los esfuerzos deseados en la búsqueda de los propósitos planteados” (Hernández-Palomino, 2017, p. 121).

\section{Aportaciones teóricas.}

\section{Diagnóstico organizacional.}

De acuerdo con Rodríguez (2016), "el diagnóstico organizacional constituye una descripción, una explicación, hecha por el observador, del operar de una organización determinada”. (p.30). Por su parte Guizar (2013), explica que "el diagnóstico consiste en la recolección de datos relativos al funcionamiento de la organización y el análisis de los mismos. A partir de ello se pueden descubrir las causas del problema o las oportunidades de la organización” (p. 197). 
El diagnóstico organizacional ha evolucionado de ser una técnica utilizada como parte del proceso de Desarrollo Organizacional (D. O.) a una técnica importante por sí misma.

\section{Cultura y cultura empresarial.}

La palabra cultura, de acuerdo con lo que establece la Real Academia Española (2018) en su diccionario, proviene del latín cultura, que significa cultivo y cuya definición es el "conjunto de modos de vida y costumbres y grado de desarrollo artístico, científico, industrial, en una época y grupo social”. Desde el punto de vista de la Sociología, Giddens (2000), define a la cultura como: "las formas de vida de los miembros de una sociedad o de sus grupos. Incluye el modo de vestir, las costumbres matrimoniales y la vida familiar, las pautas laborales, las ceremonias religiosas y los pasatiempos" (p. 21). En el contexto organizacional, la cultura empresarial de acuerdo con Mercado (2006), se precisa como: "aquellos valores e ideas comunes que, sin estar formalmente establecidos, guían el comportamiento de todos los miembros de la organización" (p. 350).

Recientemente el enfoque administrativo, de acuerdo con Chiavenato (2006), dice que "la cultura organizacional representa las normas informales y no escritas que orientan la conducta de los miembros de la organización día con día y que le dan sentido a sus acciones para la realización de los objetivos organizacionales. Cada organización tiene su propia cultura corporativa” (p. 319).

Adicionalmente Chiavenato (2006), añade que: "la única forma viable de cambiar una organización es cambiar su cultura, (...), para que las organizaciones puedan sobrevivir y desarrollarse, para que exista la renovación y la revitalización, se debe cambiar la cultura organizacional” (p. 321). Lo mencionado por Chiavenato acentúa la necesidad que debieran tener todas las organizaciones de entender su cultura y de analizarla para poder determinar así, si esta ayuda verdaderamente, por medio de su estrategia, a lograr sus objetivos.

Ritter (2008), considera a la cultura organizacional como "la manera en que actúan los integrantes de un grupo u organización y que tiene su origen en un conjunto de creencias y valores compartidos" (p. 41). Robbins y Judge (2013), por su parte, definen a la cultura organizacional como "un sistema de significado compartido por los miembros, el cual distingue a la organización de los demás" (pp. 552-553). Complementan que hay siete características que captan la esencia de la cultura de una organización: innovación y toma de riesgos, atención a los detalles, orientación a resultados, orientación a la gente, orientación a los equipos, dinamismo y estabilidad.

De acuerdo con Jones (2008), la cultura organizacional es el "conjunto de valores y normas compartidos, que controlan las interacciones entre los miembros organizacionales, así como entre estos y otras personas externas a la orga- 
nización" (p. 8). A su vez este mismo autor define a los valores como "criterios, estándares o principios generales que la gente usa para determinar los tipos de comportamiento, situaciones, eventos y resultados deseables o indeseables" (p. 8). Por su parte, Hellriegel, Scolum y Franklin, 2017), establecen que "la cultura se refiere a un patrón único de supuestos, valores y normas compartidas que dan forma a la socialización, los símbolos, el lenguaje, los relatos y las prácticas de un grupo de personas" (p. 596). Para estos autores la cultura organizacional es relevante porque tienen efecto en el rendimiento y satisfacción de los miembros.

Uno de los principales autores y pioneros en el estudio de la cultura empresarial, Edgar H. Schein (1988), la define a la cultura organizacional como:

Un modelo de presunciones básicas-inventadas, descubiertas o desarrolladas por un grupo dado al ir aprendiendo a enfrentarse con sus problemas de adaptación externa e integración interna-, que hayan ejercido la suficiente influencia como para ser consideradas válidas y, en consecuencia, ser enseñadas a los nuevos miembros como el modelo correcto de percibir, pensar y sentir esos problemas (pp. 25-26).

Zapata y Rodríguez (2008), mencionan que "la cultura corporativa se compone de diversos elementos que tienen significancia y que son compartidos por un grupo" (p.228). Estos elementos son: los valores, los símbolos, el lenguaje, los mitos y el héroe. Los valores representan la manera en cómo deberían ser las cosas, los símbolos reproducen la cultura mediante información, el lenguaje es la forma en que la organización se expresa, los mitos acogen la historia fundacional de la empresa y destacan sus valores y el héroe es el que da el ejemplo, que podría ser el fundador de la organización.

Franklin y Krieger, (2011, p. 362), mencionan las características primordiales que presenta la cultura organizacional:

1. Es holística: el todo representa más que la suma de sus partes.

2. Está históricamente determinada: refleja la historia de la organización.

3. Se construye socialmente a través de la interacción.

4. La crean y la recrean normas formales e informales que rigen la organización, los estilos de autoridad y liderazgo.

5. Es intangible, pero hace la diferencia entre una organización y otra.

6. Es difícil cambiar, pues se encuentra interiorizada en las personas, grupos, equipos, gerentes y directivos que integran la organización.

Estos mismos autores señalan que: "la cultura organizacional no puede verse de manera directa, por lo que para conocerla debemos fijarnos en una serie de indicadores y manifestaciones que caracterizan el sistema de presunciones 
básicas de la organización” (Franklin y Krieger, 2011, p. 362). Dichos indicadores son: "el clima organizacional, valores organizacionales, presunciones básicas, normas, símbolos, subculturas dentro de la organización, entorno físico y la cultura material" (Franklin y Krieger, 2011, p. 362) (Ver Figura 1).

Figura 1. Indicadores de la cultura organizacional

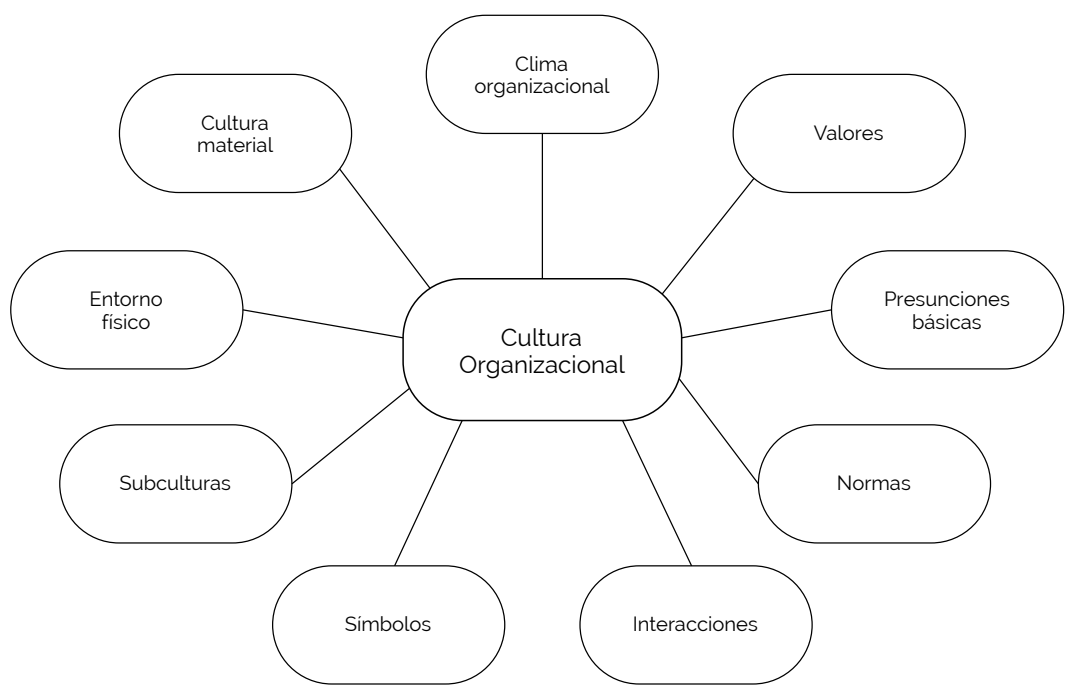

Fuente: elaboración propia con base en Franklin y Krieger (2011)

\section{Clima organizacional}

El clima organizacional es el factor más visible y se puede estudiar a través del contrato psicológico, el sistema de remuneraciones, recompensas e incentivos, el desarrollo de carrera y competencias. Análisis de las expectativas grupales, relaciones de autoridad y estilos de liderazgo, los líderes, características de la función puesto-tarea y la resolución de conflictos.

\section{Valores}

Jones (2008), define a los valores como "criterios, estándares o principios generales que la gente usa para determinar los tipos de comportamiento, situaciones, eventos y resultados deseables o indeseables" (p. 178). Los valores organizacionales son un elemento esencial de la cultura de una organización, de acuerdo con este mismo autor, los valores organizacionales se componen de los valores terminales y los valores instrumentales (Ver Figura 2). 
Figura 2. Valores organizacionales

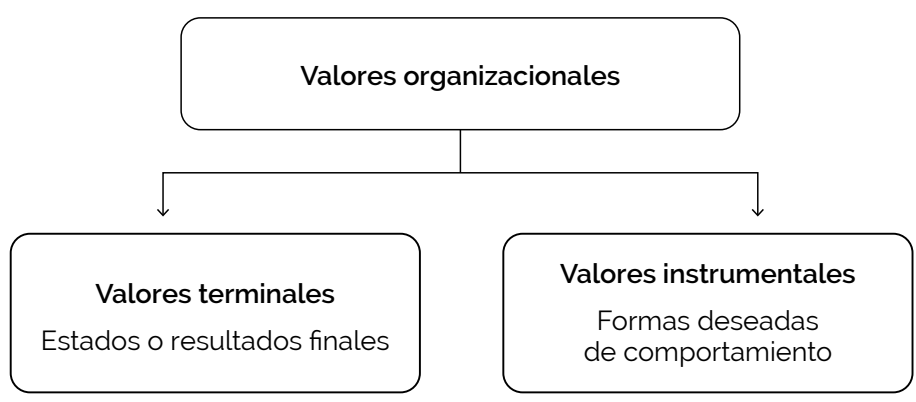

Fuente: elaboración propia con base en Jones (2013)

En la Figura 2, se expone que los valores organizacionales se componen de los valores terminales e instrumentales. Los valores terminales son los enfocados a resultados, como por ejemplo lograr una alta calidad o excelencia en sus operaciones, rentabilidad, innovación, confiabilidad. Los valores instrumentales son los medios para lograr los terminales, por ejemplo, ser útil, hacer las cosas bien y a la primera, ser honesto, tomar riesgos, etc. Por lo tanto, de acuerdo con Jones (2008), la combinación de ambos valores lleva a una cultura empresarial.

Para Arciniega y Zazueta (2010), los valores son como "las representaciones cognitivas de necesidades universales que se manifiestan a través de metas transituacionales que se organizan en forma jerárquica y se presentan en el contexto laboral” (p. 43).

\section{Presunciones básicas}

Franklin y Krieger (2011), explican que las presunciones básicas son las premisas inconscientes dentro de la empresa, que pueden ser de compromiso o no compromiso, de certeza o incertidumbre, de individualismo o de trabajo en equipo, de cuidado al ambiente de trabajo, entre otros.

\section{Normas}

Las normas, reglas o políticas aplicadas en una organización determinan también en gran medida la cultura imperante. 


\section{Interacciones}

El tipo de relación existente entre los integrantes de la organización, incluyendo empleados, socios, dirigentes, proveedores y clientes, refleja la cultura de la empresa.

\section{Símbolos}

Los símbolos se refieren a los rituales, ceremonias, lemas, e historias que se dan dentro de la organización. Estos símbolos de la cultura se desarrollan a lo largo de la vida de la empresa y en algunos casos, por ejemplo, en las historias, tienden a exagerarse; el objetivo principal de los símbolos debe ser motivar al personal a sentirse parte de la organización y alcanzar las metas y objetivos de esta.

\section{Subculturas}

Dentro de la misma cultura de la empresa existen grupos sociales con distintas formas de pensar, costumbres y valores que conviven dentro de la empresa, sin embargo, estas subculturas pueden estar opuestas a los fines estratégicos, causando fuerzas adversas a la misma.

\section{Entorno físico}

El entorno físico de las organizaciones se compone de tres elementos: estructuras físicas, estímulos físicos y objetos simbólicos. La estructura física de las instalaciones de la empresa se refiere al diseño y ubicación de las oficinas y esta determina el tipo de cultura, si los espacios son aislados y cerrados se fomentará una cultura de feudos con poca comunicación y por el contrario si los espacios son abiertos facilitaran una cultura de contacto e intercambio de ideas.

\section{Cultura material}

La cultura material se origina cuando los productos o servicios creados por las organizaciones consciente o inconscientemente reflejan las creencias de los individuos que los fabricaron o brindaron y por extensión las creencias de la sociedad a la cual pertenecen esos individuos (Franklin y Krieger, 2011, pp. 366-374).

\section{Formación de la cultura organizacional}

La formación de una cultura organizacional, la ejemplifica Robbins (2004), en el siguiente esquema (Ver Figura 3), en donde la cultura parte de la filosofía de los fundadores de la empresa que luego se transfiere a los criterios de selección de personal para integrarlos a la organización y finalmente es socializada al resto de los miembros por los directivos de esta. 
Figura 3. Cómo se forma la cultura de una organización

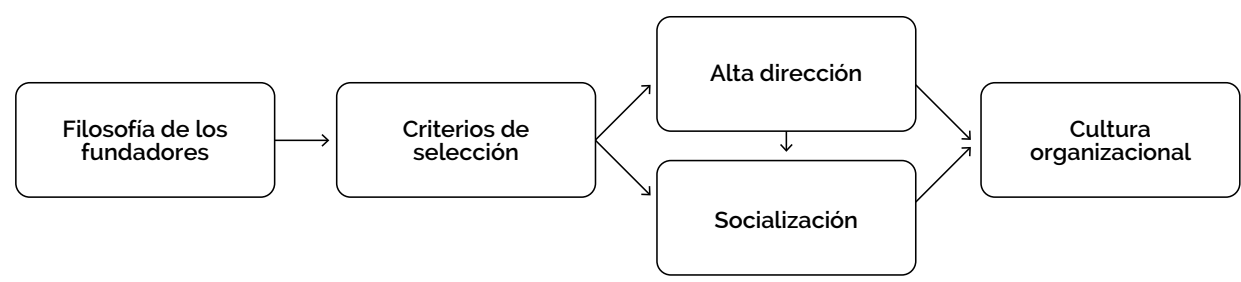

Fuente: elaboración propia basada en Robbins (2004)

\section{Clasificación de la cultura empresarial}

Robbins (2004), clasifica la cultura empresarial en virtud de su trascendencia o calado en fuerte, cultura en la que los valores centrales son sostenidos con firmeza y son ampliamente compartidos y débil, cultura en donde no se asumen los valores, sino que se conciben como impuestos. Según este mismo autor, el efecto que tienen las culturas empresariales en el desempeño de la organización depende en gran medida de la fortaleza en dicha cultura, entre más fuerte sea, mayor será el desempeño operacional y viceversa. (Ver Figura 4).

Figura 4. Efecto de las culturas organizacionales en el desempeño y la satisfacción

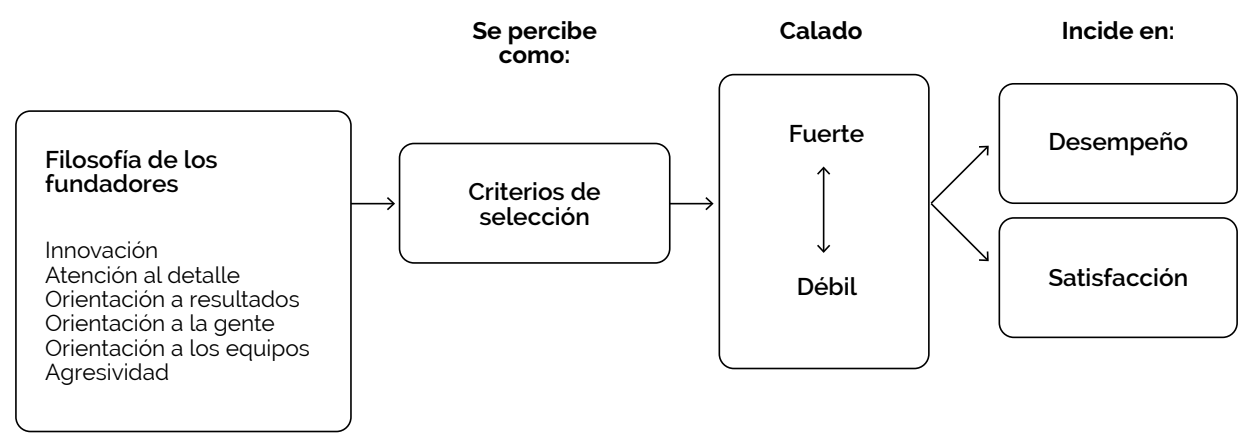

Fuente: elaboración propia basada en Robbins (2004) 
Cuanto más fuerte sea la cultura de una organización, menos necesidad tendrá la gerencia de preocuparse por desarrollar reglas y directrices formales que guíen la conducta de los empleados, quienes internalizan esa normatividad cuando aceptan la cultura de la organización (Robbins y Judge, 2013).

De acuerdo con Hellriegel et al. (2017), para construir una cultura fuerte se necesita que sus elementos visibles proyecten un solo mensaje consistente, donde los gerentes y empleados comparten un mismo estilo de comportamiento, utilizan un mismo enfoque para resolver problemas, cumplir metas y tratar a los clientes, proveedores y otros grupos de interés. Y agregan "cuando el entorno de negocios de una organización es relativamente estable, las culturas fuertes que apoyan metas estratégicas contribuyen al desempeño de la empresa” (p. 606).

\section{¿Cómo entender entonces a la cultura empresarial?}

Para efectos del presente artículo, con base en las aportaciones teóricas revisadas, se entenderá a la cultura empresarial, como el conjunto de creencias, valores, símbolos y costumbres que definen los comportamientos compartidos por los integrantes de una organización, aprendidos de aciertos y errores del pasado, y que a su vez impacta con base en la estrategia definida por los gerentes, en los resultados de la empresa (Ver Figura 5).

Figura 5. La cultura empresarial como elemento de impacto en los resultados organizacionales

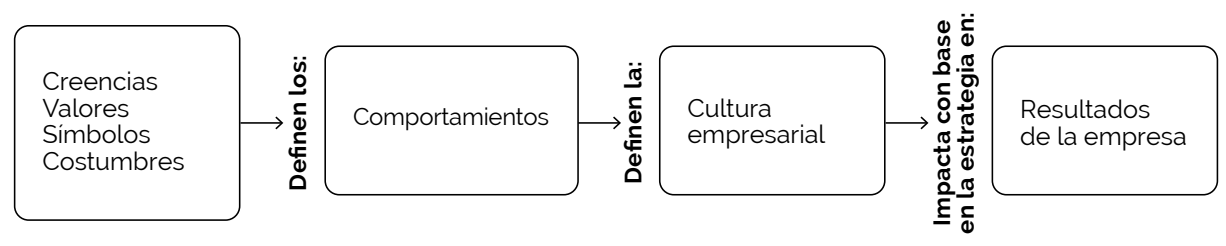

Fuente: elaboración propia 


\section{Cultura empresarial en México}

De acuerdo con David (2003), México es una sociedad autoritaria y se puede ver en varias organizaciones, como escuelas, iglesias, empresas y familias. Los empleadores "buscan trabajadores que sean agradables, respetuosos y obedientes, más que innovadores, creativos e independientes. Los trabajadores mexicanos tienden a orientarse a la actividad y menos a la solución de problemas" (p. 258).

Rodríguez-Valencia (2002), en su libro Administración de pequeñas y medianas empresas, afirma que el familismo es una fuerza que influye en el comportamiento empresarial de nuestro país y que está inmerso en la cultura mexicana (p. 107), y que además es uno de los valores más comunes en los países en vías de desarrollo en donde se le da gran importancia al parentesco entre los miembros de las organizaciones empresariales.

"La cultura define el color de como vemos el mundo" (Hellriegel, et al. 2017, p. 606). Estos autores tratan de transmitir a través de esta frase la complejidad de los valores culturales que conforman a una organización o país y explican las diferencias entre los valores y las características gerenciales entre México y Estados Unidos. Por ejemplo, de acuerdo con estos autores, los mexicanos prefieren que el responsable de la toma las decisiones este centralizada, mientras que en Estados Unidos se prefiere la participación y delegación en el proceso de toma de decisiones; en el caso del proceso de planeación, en Estados Unidos la planeación es formal, proactiva y de largo plazo, mientras que en México es no estructurada, reactiva y a corto plazo.

\section{Cambio cultural}

Las organizaciones están inmersas en un entorno cambiante que obligan a las empresas a modificar sus estructuras, según Hernández-Palomino (2017), el enfoque anterior de integración vertical tiene que cambiar hacia el "uso de alianzas, asociaciones y empresas en participación” (pp.134-135). En el actual entorno en donde los cambios son la constante, las organizaciones deben de ser flexibles para poderse adaptar, por lo tanto, es importante contar con una cultura proactiva y no reactiva, es decir prepararse anticipadamente y no reaccionar cuando la situación ya cambió.

Es por lo anterior que las organizaciones están obligadas ante el cambio forzoso y drástico dado la enorme competencia y las exigencias de los clientes, en adelantos tecnológicos y avances en las distintas ciencias para sobrevivir y crecer en el mercado y añade: "como los cambios suelen ser profundos en quienes lo sufren, toda organización tiende a verse afectada en alguna de sus partes 
por el cambio” (Hernández-Palomino, 2017, p.135). Sin embargo, en la mayoría de las pymes mexicanas la resistencia al cambio es altamente frecuente, por lo que, para sobrevivir, forzosamente las empresas necesitan hacer cambios en su estructura y procesos para adecuarlos al contexto actual. Según Hernández-Palomino (2017), explica que "existen cinco técnicas que pueden utilizarse para lograr un proceso de cambio eficaz” (p. 131), las cuales son:

1. Entrenamiento en sensibilización. Esta se refiere a un método para cambiar el comportamiento por medio de una interacción no estructurada

2. Retroalimentacion de encuestas. Se evalúan las actitudes y se identifican las discrepancias entre los miembros de una organización por medio de encuestas

3. Consultoría en proceso. Esta técnica recurre a un consultor externo que le dan al cliente el conocimiento de lo que pasa alrededor de él, dentro de él y entre el cliente y la demás gente

4. Integracion de equipos. Esta técnica utiliza actividades de grupo de alta interacción para incrementar confianza y apertura entre los miembros de un equipo

5. Desarrollo intergrupal. Busca cambiar las actitudes, los estereotipos y las percepciones de los grupos entre sí (Hernández-Palomino, 2017, pp.131-133).

\section{Metodología}

Esta investigación se realizó con un enfoque cuantitativo; el diseño de la investigación, de acuerdo con Hernández-Sampieri, Fernández-Collado y Baptista-Lucio (2014), es de tipo cuasi experimental transeccional y exploratoria. Es cuasi experimental, porque se llevó a cabo sin manipular deliberadamente variables y se basó en conceptos, comunidades o contextos que ya ocurrieron. Transeccional exploratorio, porque se comienza a conocer una variable, un evento, una situación, y es una exploración inicial dado que se aplica a problemas de investigación poco estudiados que constituyen el preámbulo de otros diseños de investigación.

Para este estudio, se consideró una población de 35 pequeñas y medianas empresas del sector privado, localizadas y originarias del municipio de Tamazula de Gordiano, Jalisco, México, obtenida de la base de datos del Directorio Estadístico Nacional de Unidades Económicas 2018, D.E.N.U.E. (INEGI, 2018). De estas, 17 correspondieron al sector de servicios, 3 al de producción y 15 al comercial (Ver Figura 6). 
Figura 6. Población analizada

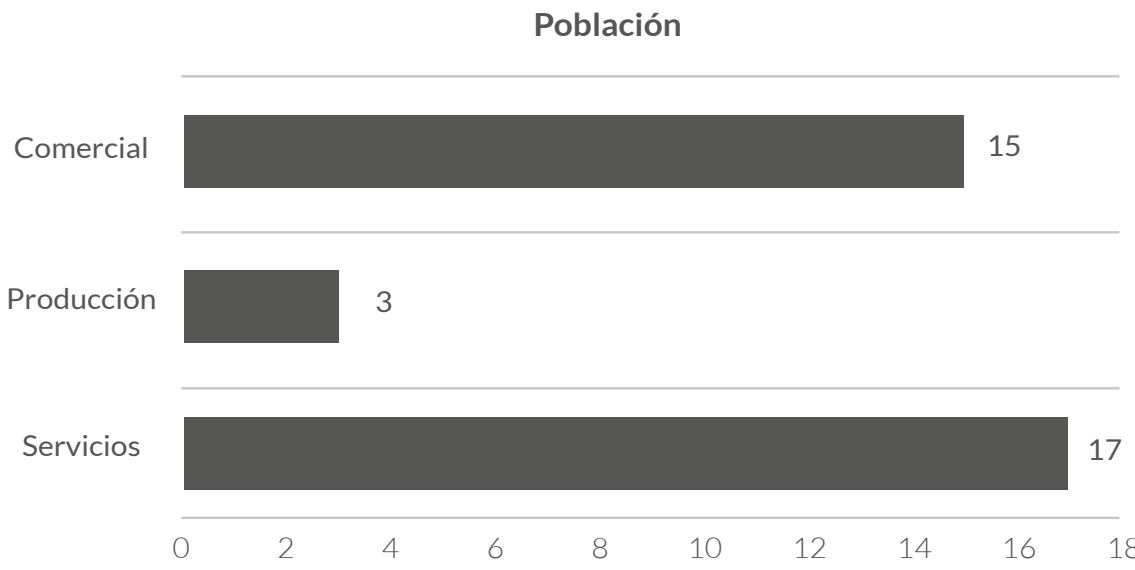

Fuente: elaboración propia basada en datos del INEGI (2018)

El tamaño de las empresas seleccionadas se determinó considerando la información publicada en el Diario Oficial de la Federación del 30 de junio de 2009, citada en el documento Censos Económicos (INEGI, 2011) Micro, pequeña, mediana y gran empresa, en donde se precisa que se consideran pequeñas y medianas empresas aquellas que cuentan de entre 11 a 250 empleados en el sector producción, mientras que de 11 a 100 en el comercial y de servicios (Ver Tabla 1).

Tabla 1. Clasificación de las empresas por número de trabajadores

\begin{tabular}{c|c|c|c}
\multicolumn{5}{c}{ Clasificación por número de trabajadores } \\
\hline Sector / tamaño & Producción & Comercial & Servicios \\
\hline Micro empresa & $0-10$ & $0-10$ & $0-10$ \\
\hline Pequeña empresa & $11-50$ & $11-30$ & $11-50$ \\
\hline Mediana empresa & $51-250$ & $31-100$ & $51-100$ \\
\hline Gran empresa & A partir de 251 & A partir de 101 & A partir de 101 \\
\hline
\end{tabular}

Fuente: elaboración propia con datos obtenidos de INEGI (2011) 
Para la recopilación de datos se utilizó como instrumento el “diagnóstico empresarial” desarrollado por el Instituto para el Desarrollo y la Innovación en la Tecnología para la Pequeña y Mediana Empresa (IDITpyme, 2014) de la Universidad de Guadalajara, considerando el área de cultura empresarial. Este diagnóstico "permite ponderar a través de varios ítems las diversas áreas funcionales de la empresa, en relación a prácticas empresariales de clase mundial y prácticas que cohíben el crecimiento de la misma” (IDITpyme, 2014).

Las áreas consideradas en el instrumento son: cultura empresarial, administración, servicio, comercialización y mercadotecnia, finanzas, recursos humanos e innovación y desarrollo tecnológico. Para el caso de la presente investigación se diagnosticó y analizó el área de cultura empresarial. El análisis de los datos recabados se realizó utilizando una hoja de cálculo (Ver Tabla 2).

Tabla 2. cha técnica de la investigación

\begin{tabular}{c|c} 
Características & Encuesta \\
\hline Población & 35 \\
\hline Ámbito de estudio & $\begin{array}{c}\text { Pequeñas y medianas empresas privadas originarias } \\
\text { y localizadas en el municipio de Tamazula de Gordia- } \\
\text { no, Jalisco, México }\end{array}$ \\
\hline Unidad & Empresa categorizada como pyme \\
\hline Recolección de información & $\begin{array}{c}\text { Entrevista mediante instrumento de diagnóstico } \\
\text { aplicado al gerente / dueño de la empresa }\end{array}$ \\
\hline Fecha del trabajo de campo & Mayo 2018 \\
\hline
\end{tabular}

Fuente: elaboración propia.

\section{Resultados}

Los resultados obtenidos del análisis de los datos producto de la aplicación del instrumento: cuestionario de diagnóstico empresarial (IDITpyme, 2014), de la Universidad de Guadalajara en el área de cultura empresarial, donde se entrevistaron a los dueños/gerentes de 35 pymes ubicadas y originarias del municipio de Tamazula de Gordiano, México, se presentan a continuación. 
Figura 7. Relación entre capital de la empresa y el patrimonio familiar
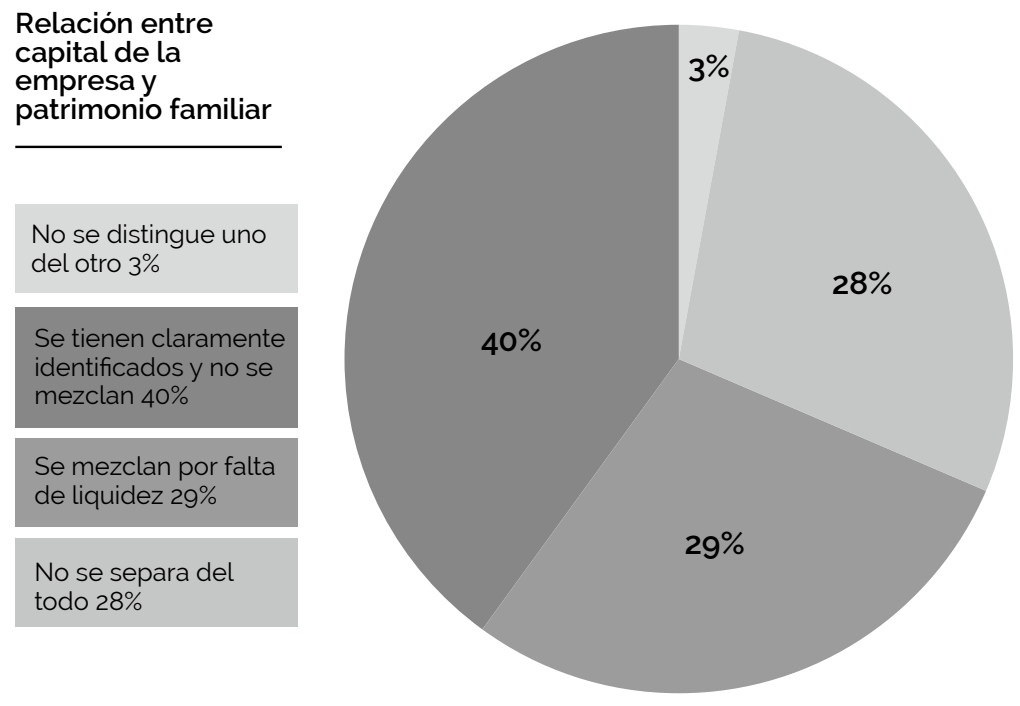

Fuente: elaboración propia

En el 29\% de las pymes encuestadas se encontró que el patrimonio de la empresa se mezcla con el familiar debido a la falta de liquidez, y por lo tanto el efectivo propiedad de la familia hace las veces de una fuente de financiamiento aparentemente sin costo para la empresa; el otro $28 \%$ contestó que no se separa del todo y el restante $3 \%$ no distingue uno del otro. El diferencial del $40 \%$, contestó tener claramente identificados ambos conceptos sin mezclarlos (Ver Figura 7).

Con estos datos se evidencia que el $60 \%$ de las pymes del municipio de Tamazula, no tienen dentro de su cultura, el mantener los bienes de la empresa y los familiares por separado, siendo esta práctica común entre las pymes mexicanas. El capital de la empresa y el patrimonio de la familia se combinan de forma desordenada haciendo uso de activos personales o de la familia, como por ejemplo de la casa, el efectivo, los automóviles, los terrenos, etc., considerando a esta como un apoyo temporal que a veces se vuelve indeterminado.

Las razones que los Gerentes encuestados dieron acerca de esta práctica fueron en su mayoría las siguientes: 
1. El dueño o gerente considera que como siempre se han hecho las cosas de una misma manera, y ha funcionado, se tienen que continuar haciendo de la misma forma.

2. Los familiares involucrados en las pymes, consideran que tienen derechos sobre las propiedades de la empresa y los pueden utilizar a discreción.

3. Se realizan acuerdos verbales y estos no se documentan, originando malos entendidos entre los mismos familiares respecto al uso de los bienes de la empresa o de la familia.

Considerando los puntos anteriores, es importante que las pymes documenten los acuerdos verbales que tengan que ver con el uso de activos de la familia y de la empresa, que se cumplan y tengan una temporalidad. Adicionalmente, es prioritario promover una cultura al interior de la familia que influya para que se separen los bienes de la misma y los de la empresa, logrando así una administración formal del patrimonio, evitando conflictos familiares que pudieran poner en riesgo el desempeño de la organización.

Figura 8. Filosofía organizacional

\section{Filosofía Organizacional}

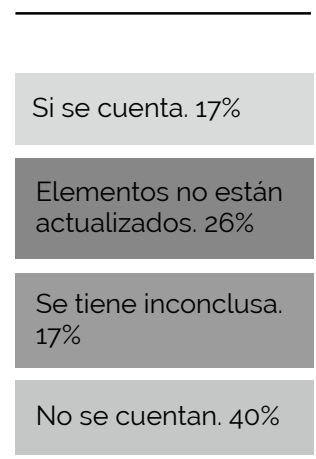

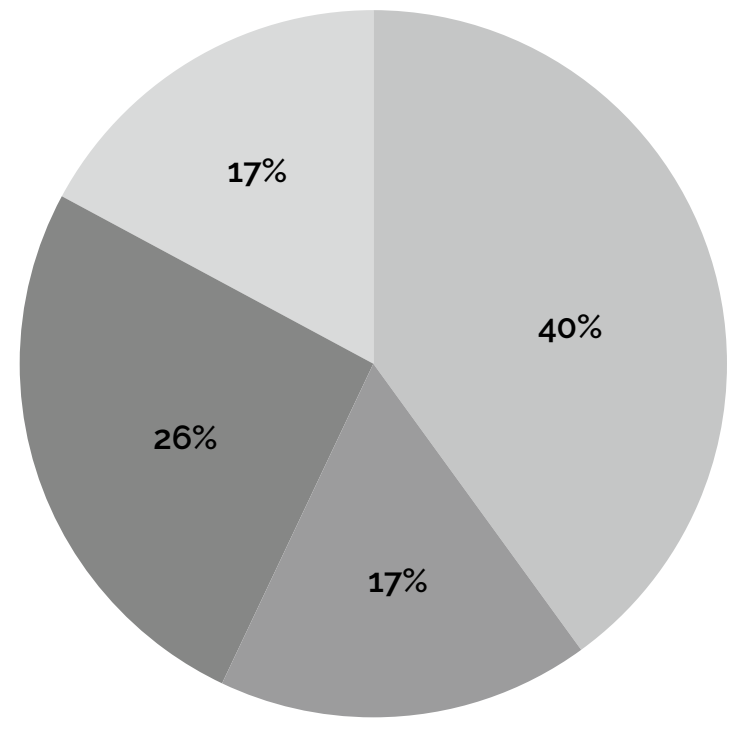

Fuente: elaboración propia 
Respecto al análisis de la filosofía organizacional, los resultados (ver figura 10) muestran que únicamente el $17 \%$ de las empresas encuestadas cuenta con una filosofía bien definida. El 40\% no cuenta con ella, el 26\% dijo que sus elementos están desactualizados y el $17 \%$ restante manifestó que esta inconclusa.

La filosofía organizacional de acuerdo con Pérez (2008), "trata los valores, las convicciones filosóficas de los gerentes (...); misión, el concepto general de su empresa; visión, cómo debe ser su empresa en el futuro, y estrategia, la dirección en que debe avanzar su empresa" (p. 12). De acuerdo con Koontz y Weihrich (2013), una de las características de las empresas de excelencia es el que "las impulsa una filosofía de la compañía basada en los valores de sus líderes” (p. 7).

Así pues, podemos definir a la filosofía corporativa como una edificación ideada desde dentro de la organización, externalizada a través de los principios, valores, misión y la visión que la integran. Las pymes de Tamazula, al no contar con una declaración de misión o razón de ser de la empresa, una visión o dirección que muestre hacia donde se quiere llegar y sin valores determinados que deben definir los comportamientos de sus integrantes, quedan en una posición claramente desventajosa con relación a sus competidores de mayor tamaño que tienen una filosofía bien estructurada y compartida por sus miembros.

Figura 9. ¿El personal conoce la filosofía?
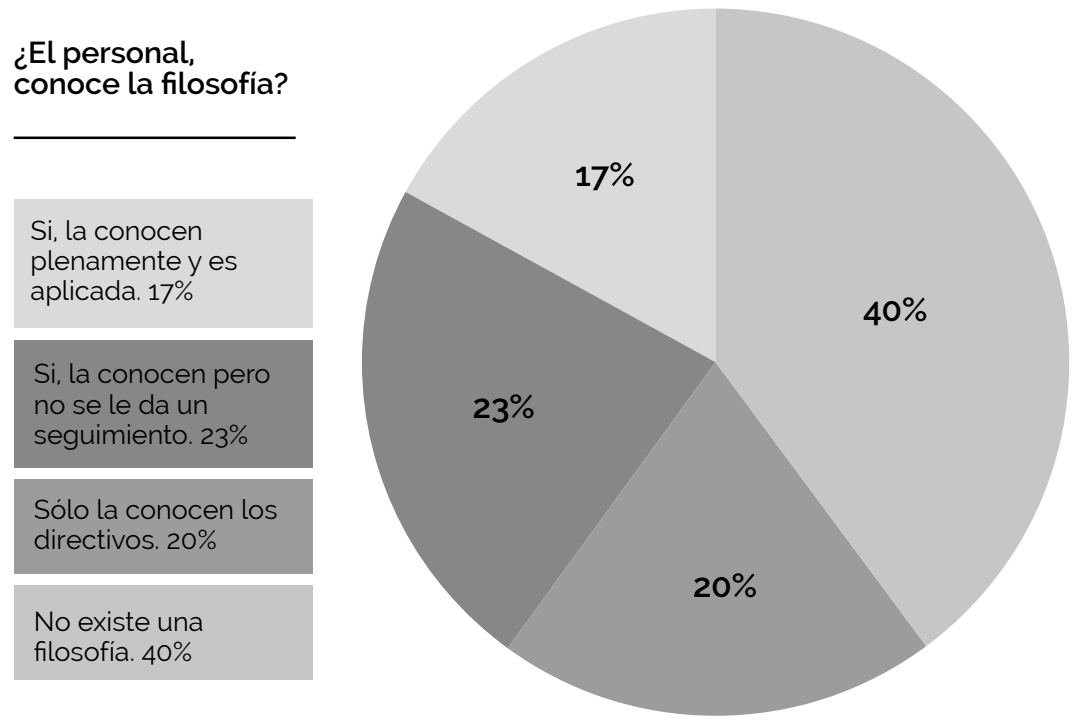

Fuente: elaboración propia 
En relación con la pregunta de si el personal conoce la filosofía de la empresa, el 40\% de las pymes de Tamazula contestó que no cuentan con una filosofía organizacional, el 20\% solamente la conocen los directivos o dueños y el $23 \%$ si la conocen, pero no se le da seguimiento. Únicamente el 17\% cuenta con una, que es conocida por los integrantes de la organización y es aplicada plenamente (Ver Figura 9).

En suma, el $83 \%$ de las pymes no cuentan con una filosofía o solamente la conocen sus dueños o gerentes y no se le da seguimiento, por lo que se infiere, que el hecho de contar con principios y valores en las pequeñas y medianas empresas de Tamazula, no son una prioridad, ocasionando disparidad entre los valores que los dueños tienen, con los que su personal acepta, pudiendo originar problemas como la rotación de personas y la necesidad de implementar reglamentos estrictos para intentar disminuir posibles conflictos conductuales entre sus miembros.

La filosofía corporativa, de acuerdo con Viñarás, Cabezuelo y De la Casa Herranz, 2015) está compuesta por tres aspectos básicos: la misión, los valores y la visión corporativa (pp. 395-396). Los valores como parte de la filosofía de la organización, según Arciniega y Zazueta (2010), son "las representaciones cognitivas de necesidades universales que se manifiestan a través de metas transituacionales que se organizan en forma jerárquica y se presentan en el contexto laboral” (p. 35). Entre más miembros conozcan y acepten los valores fundamentales de la organización y mayor sea su compromiso más fuerte será la cultura. Una cultura fuerte debería de reducir la rotación de personal, ya que demuestra un alto nivel de acuerdo con aquello que la organización representa.

Cuanto más fuerte sea la cultura de una organización, menos necesidad tendrá la gerencia de preocuparse por desarrollar reglas y directrices formales que guíen la conducta de los empleados, quienes internalizan esa normatividad cuando aceptan la cultura de la organización (Robbins y Judge, 2013). Complementariamente, Hernández-Palomino (2017), menciona que una misión clara puede ser un buen motivador para los empleados. La misión debe de estar pensada en el crecimiento de la empresa, ya que a medida que las organizaciones crecen, la comunicación directa con el personal se hace más difícil y no se pueden trasmitir los planes, la visión y los valores que regirán la empresa a futuro tan fácilmente. 
Figura 10. ¿Practica alianzas estratégicas?
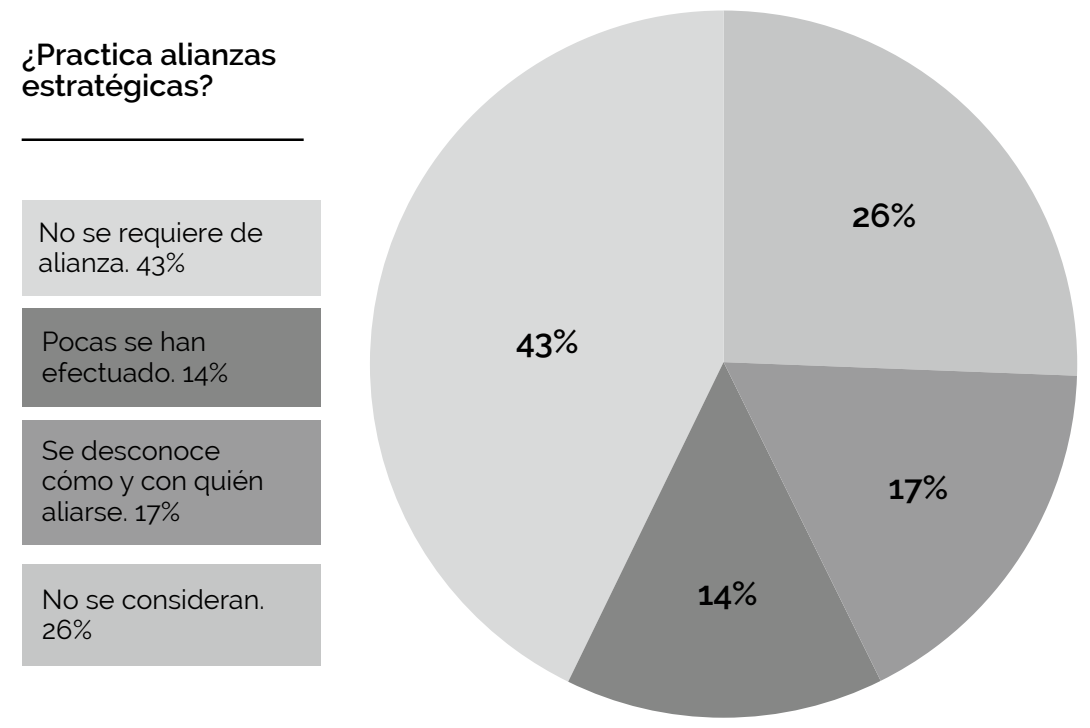

Fuente: elaboración propia

En relación con la práctica de alianzas estratégicas, 43\% de las empresas encuestadas contestó que no se requiere de estas para que la pyme mejore sus resultados; el 26\% dijo que no se consideran necesarias y otro $17 \%$ mencionó que desconoce con quien aliarse. Únicamente el 14\% reconoce haber efectuado algún tipo de alianza (Ver Figura10). Con estos datos se pueden establecer los siguientes tres puntos:

1. El $86 \%$ de las pymes desconoce qué es y para qué sirven las alianzas estratégicas.

2. De las empresas que conocen el término, el $43 \%$ considera que no son necesarias para expandir o mejorar en algún sentido a su empresa.

3. Únicamente el $14 \%$ de las pymes, admite haber realizado algún tipo de alianza, sin necesariamente ser de carácter estratégico.

Según Johnson, Scholes y Whittington (2006) “una alianza estratégica se produce cuando dos o más organizaciones comparten recursos y actividades para practicar una determinada estrategia” (p. 349). Las alianzas estratégicas 
son de suma importancia para que las pymes logren incrementar sus ventajas frente a sus competidores, ya que se producen sinergias y nuevos enfoques en el modo de explorar e introducirse a mercados potenciales.

Mercado (2006), agrega que las alianzas estratégicas es una tendencia mundial, la cuales "permiten incrementar las ventajas de cada una de las partes al producirse sinergias de importancia y nuevos enfoques en el modo de atacar los diferentes mercados” (p. 347).

Actualmente las organizaciones están inmersas en un entorno cambiante que obliga a la empresa a modificar sus estructuras, según Hernández-Palomino (2017), el enfoque anterior de integración vertical, por ejemplo, tiene que cambiar hacia el "uso de alianzas, asociaciones y empresas en participación" (p.134135). El no conocer los beneficios de realizar este tipo de alianzas limita el desarrollo de las pymes e incrementa la probabilidad de perder oportunidades de negocio a futuro.

Figura 11. Postura de directivos ante problemas

\section{Postura de directivos ante problemas}

Se anticipan problemáticas con postura pro activa. $6 \%$

Se diagnostican a tiempo y se enfrentan de forma reactiva. 20\%

Se actúa en consecuencia (a posteriori). 37\%

Se detectan una vez que suceden, se actua de forma reactiva. $37 \%$

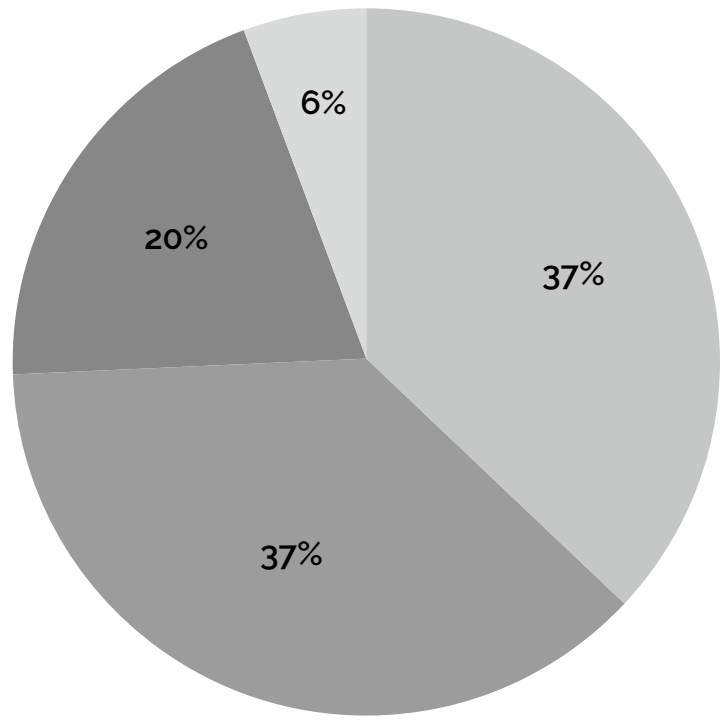

Fuente: elaboración propia 
Respecto a la postura que tienen los directivos ante problemas que se les presentan, (Ver Figura 11), se observa que el 37\% de los gerentes/ dueños encuestados, tienen una posición claramente reactiva, otro $37 \%$ detecta los problemas y actúa a posteriori, el $20 \%$ detecta a tiempo los problemas, pero actúa de manera reactiva también y solamente el $6 \%$ manifestó anticiparse a la problemática con una postura proactiva.

El resultado sugiere que la cultura de los directivos de las pymes de Tamazula, es de tipo reactivo, no planificada, de vivir al día sin ser capaces de visualizar escenarios que pongan en riesgo la continuidad de la empresa, y por ende, sin las estrategias para enfrentarlas.

En el actual entorno en donde los cambios son la constante, las organizaciones deben de ser flexibles para poderse adaptar (Hernández-Palomino, 2017), por lo tanto, es importante contar con una cultura proactiva y no reactiva, es decir prepararse anticipadamente y no reaccionar cuando la situación ya cambió.

Dado lo anterior es importante que las pymes elaboren una planeación estratégica, la cual se refiere a la "formulación de estrategias para aprovechar oportunidades nuevas y diferentes para el futuro" (David, 2017, p. 5). La planeación estratégica ayuda a los gerentes a identificar oportunidades y amenazas y considerarlas para formular la misión, planes y estrategias para la empresa, formulando planes para los continuos cambios en los entornos de sus negocios. La planeación estratégica de una empresa de acuerdo con Michel Porter (citado por Hellriegel et al., 2017), debe incluir una evaluación de las cinco fuerzas competitivas: competidores, nuevos entrantes, clientes, proveedores y bienes y servicios sustitutos (pp. 232-234).

\section{Conclusiones}

La cultura empresarial es un elemento distintivo de cualquier organización; es su forma de ser y de actuar ante determinados acontecimientos; es una cultura aprendida de aciertos y errores cometidos en el pasado, formada y transmitida por sus propietarios. La cultura de las pymes localizadas y originarias del municipio de Tamazula de Gordiano, se caracteriza por no distinguir o separar el capital de la empresa y el patrimonio familiar, porque solamente una minoría de las empresas cuenta con una filosofía organizacional bien definida y conocida por sus miembros, en donde la mayoría de los gerentes o dueños consideran a las alianzas estratégicas como de poca o sin importancia alguna para el logro de los objetivos organizacionales y con una posición reactiva de sus gerentes o dueños frente a posibles amenazas. 
El que las pymes carezcan en su mayoría de una filosofía organizacional bien definida, compartida y transmitida a sus integrantes, en un medio altamente competitivo y que a pesar de ello siguen apegadas a los mismos valores y modos de conducción tradicionales, son razones que "alertan acerca de que algo se debe hacer para cambiar la cultura de la organización” (Franklin y Krieger, 2011, p. 380).

Por otra parte, se infiere que, dadas las características examinadas, la cultura empresarial de las pymes de Tamazula, es una cultura débil, de acuerdo con la clasificación de Robbins (2004), lo que pone a las pequeñas y medianas empresas en una situación de desventaja frente a las grandes organizaciones que se caracterizan por contar con una cultura fuerte que les permite tener un mejor desempeño y ser más competitivas en un mercado extremadamente demandante. Esta cultura débil pone a las pymes de Tamazula en una situación de riesgo de permanencia ante una situación económica local y nacional cada vez más compleja, con la llegada de competidores como cadenas regionales o nacionales que han venido estableciéndose en este municipio mexicano durante la última década.

Finalmente, se concluye que las características de la débil cultura empresarial que predomina en las pymes del municipio de Tamazula de Gordiano, son similares en las pequeñas empresas de los distintos municipios en México y otros países de Latinoamérica, debido a las grandes similitudes culturales que coexisten y se comparten entre los países de esta región del mundo.

\section{Recomendaciones}

Es importante que las pymes del municipio de Tamazula de Gordiano separen el capital de la empresa del patrimonio familiar, no mezclándolos entre sí, con la finalidad de establecer una cultura en donde se priorice una administración profesional que permita la clara identificación de los activos propiedad de la empresa y de la familia, evitando con esto problemas internos y fomentando una cultura de transparencia que marque los límites entre los activos mencionados, con la finalidad de mejorar su desempeño.

También es imprescindible elaborar y/o actualizar la filosofía organizacional, que incluya al menos la misión, visión, y valores. Una vez elaborada es necesario darla a conocer a todo el personal de la empresa. La difusión y sensibilización de los valores empresariales son tareas primordiales para cualquier organización, debido a que influyen directamente en el proceso de dotación de capital humano, si los nuevos integrantes no comparten dichos valores, difícil será su integración a la empresa, causando conflictos entre el personal de la misma al actuar guiados por valores distintos o no permitidos por la gerencia de la empresa. 
Existe, además, la oportunidad de establecer alianzas estratégicas que puedan ayudar a las pymes de Tamazula a lograr el acceso a nuevos mercados, mejor tecnología e investigación aplicada, mediante fabricantes, proveedores y centros de estudio entre otros. Finalmente, resulta prioritario para las pymes del municipio de Tamazula de Gordiano, Jalisco (México), realizar una adecuada planificación estratégica que les permita, anticipar amenazas que pongan en riesgo la permanencia de la empresa con una actitud proactiva centrada en la mejora continua, buscando el logro de los objetivos y metas organizacionales.

\section{Agradecimientos}

Reconociendo toda responsabilidad por cualquier omisión que aún subsista, los autores agradecen atentamente los comentarios de los dictaminadores anónimos, cuyas contribuciones permitieron mejorar sustancialmente este trabajo.

\section{Referencias}

Arciniega L.; Zazueta, H. (2010). Desarrollo de valores en el trabajo. México, D. F.: Trillas.

Benavides-Pañeda, J. (2004). Administración: México, D. F.: McGraw-Hill.

Chiavenato, I. (2006). Introducción a la teoría general de la administración. México, D. F.: McGraw-Hill Interamericana.

David, F. (2003). Conceptos de Administración Estratégica. México, D. F.: Pearson Educación.

David, F. (2017). Conceptos de Administración Estratégica. México, D. F.: Pearson.

Franklin, B.; Krieger, M. (2011). Comportamiento Organizacional. Enfoque para América Latina. México, D. F.: Pearson educación.

Franklin, B. (2014). Organización de empresas. México, D. F.: McGraw-Hill. Interamericana editores, S.A. de C.V. 
Garza-Treviño, J. (2004). Valores para el ejercicio profesional. México, D. F.: McGraw-Hill.

Giddens, A. (2000), Sociología. Madrid, España: Alianza Editorial.

Gobierno de Jalisco (2019). Municipio Tamazula de Gordiano. https://www.jalisco.gob.mx/wx/jalisco/municipios/tamazula-de-gordiano [Consultado el 12 de septiembre de 2019 ].

Gómez R., J.; Villarreal S., M.; Villarreal, S. F. (2014). La cultura empresarial y su relación con los estilos de aprendizaje en la industria de celulosa, cartón y papel en México. Investigación Administrativa, 113, 7-27. http://www.redalyc.org/articulo.oa?id=45604495600 1

[Consultado el 11 de octubre de 2019].

Guizar, R. (2013). Desarrollo Organizacional. Principios y aplicaciones. México, D. F.: McGraw-Hill.

Hellriegel, D.; Jackson S.; Scolum, J.; Franklin, E. (2017). Administración: un enfoque basado en competencias. México, D. F.: Cengage Learning Editores, S.A. de C.V.

Hernández-Palomino, J. (2017). Desarrollo Organizacional. Teoría, práctica y casos. México, D. F.: Pearson.

Hernández-Sampieri, R.; Fernández-Collado, C.; Baptista-Lucio, P. (2014). Metodología de la investigación. México, D. F.: McGraw-Hill / Interamericana editores S.A.

Instituto Nacional de Estadística y Geografía, INEGI. (2000). Directorio Estadístico Nacional de Unidades Económicas (DENUE). https://www.inegi.org.mx/temas/directorio/

[Consultado el 25 de marzo de 2018].

Instituto Nacional de Estadística y Geografía, INEGI. (2011). Micro, pequeña, mediana y gran empresa: estratificación de los establecimientos: Censos Económicos, 2009, 1-2. https://www.inegi.org.mx/contenidos/programas/ce/2009/doc/minimonografias/m_pymes.pdf [Consultado el 20 de febrero de 2018 ]. 
Instituto Nacional de Estadística y Geografía, INEGI. (2015). Encuesta Nacional sobre Productividad y Competitividad de las Micro, Pequeñas y Medianas Empresas (ENAPROCE). http://www.inegi.org.mx/est/contenidos/Proyectos/encuestas/establecimientos/otras/enaproce/default_t.aspx [Consultado el 7 de marzo de 2018].

Instituto Nacional de Estadística y Geografía, INEGI. (2018). Directorio Estadístico Nacional de Unidades Económicas (DENUE).

https://www.inegi.org.mx/app/descarga/?ti=6

[Consultado el 20 de febrero de 2018].

Instituto para el Desarrollo de la Innovación y Tecnología de la pyme, IDITpyme. (2014). Metodología para el diagnóstico de gestión empresarial. http:// iditpyme.cucea.udg.mx/sites/iditpyme/files/metodologia_iditpyme.pdf [Consultado el 14 de marzo de 2018].

Johnson, G.; Scholes, K.; Whittington, R. (2006). Dirección estratégica. Madrid, España: Pearson Educación, S.A.

Jones, G. (2008). Teoría organizacional. Diseño y cambio en las organizaciones. México. D. F.: Pearson Educación.

Jones, G. (2013). Teoría organizacional, diseño y cambio en las organizaciones, México, D. F.: Pearson educación.

Koontz, H.; Weihrich, H. (2013). Elementos de administración. Un enfoque internacional y de innovación. México, D. F.: McGraw-Hill/Interamericana editores S.A. de C.V.

Marulanda, C.; López M.; Castellanos, J. (2016). La cultura organizacional y su influencia en las buenas prácticas para la gestión del conocimiento en las Pymes de Colombia. AD-minister, 29, 163-176. http://www.redalyc.org/ articulo.oa?id=322348399008 [Consultado el 19 de noviembre de 2019].

Mercado, S. (2006). Administración de pequeñas y medianas empresas. (Estrategias de crecimiento). México, D. F: Publicaciones Administrativas Contables Jurídicas, S.A. de C.V.

Pérez, C. (2008). Pensamiento Estratégico. Fundamentos del pensamiento estratégico. Material de apoyo. Maracaibo, Venezuela: Universidad Rafael Belloso Chacín. 
Ritter, M. (2008). Cultura Organizacional: gestión y comunicación. Buenos Aires, Argentina: La Crujía.

Robbins, S. (2004). Comportamiento Organizacional. México, D. F.: Pearson Educación.

Robbins, S.; Judge, T. (2009.) Comportamiento Organizacional. México, D. F.: Pearson.

Robbins, S.; Judge, T. (2013). Comportamiento organizacional. México, D. F.: Pearson.

Rodríguez, D. (2016). Diagnóstico Organizacional. México, D. F.: Alfaomega.

Rodríguez-Valencia, J. (2002). Administración de pequeñas y medianas empresas. México, D. F.: International Thomson Editores S. A. de C.V.

Schein, E. (1988). La cultura empresarial y el liderazgo. Una visión dinámica. Barcelona, España: Plaza \& Janes Editores.

Secretaría de Economía (2010). México Emprende.

http://www.2006-2012.economia.gob.mx/mexico-emprende/empresas [Consultado el 10 de julio de 2019].

Viñarás, M.; Cabezuelo L.; De la Casa Herranz, J. (2015). Filosofía corporativa y valores de marca como ejes del nuevo paradigma comunicativo. Prisma Social, 14, 379-410.

https://www.redalyc.org/pdf/3537/353744530012.pdf

[Consultado el 13 de noviembre de 2019].

Zapata A.; Rodríguez, A. (2008). Cultura organizacional. Cali, Colombia: Universidad del Valle -Facultad de Ciencias de la Administración. 